Result. The questionnaire for the medical professionals gained 62 respondents and the one for the general population had 122 respondents, with responses from multiple nations. Overall, COVID-19 has affected everyone's mental health to a degree, and all groups had reservations about disclosing their mental health issues to others. The medical professionals were especially reluctant to disclose mental illness to their patients, but were more comfortable when it came to disclosing mental illness to colleagues. The general population, however, was much more reluctant to disclose mental health issues to their colleagues. The general population were, on the whole, willing to listen to and help anyone who came to them with mental health concerns. Both groups surveyed showed reluctance toward disclosure to the wider community.

Conclusion. COVID-19 appears to significantly affect not only physical health, but mental health as well. There is at least some degree of stigma surrounding the disclosure of mental health issues. While most would be happy to help anyone who came to them with their mental health problems, there seems to be an attitude shift when people must contend with mental health issues of their own.

\section{Tolerability of a single IV administration of a methylene blue challenge in patients with bipolar disorder: preliminary data from a pharmaco-MRI study}

Harriet Sharp ${ }^{*}$, Alfonso Russo, Alessandro Colasanti, Antonello Pinna, Prince Nwaubani and Riccardo De Marco Department of Clinical Neuroscience and Neuroimaging, Brighton and Sussex Medical School, Sussex Partnership NHS Foundation Trust

${ }^{*}$ Corresponding author.

doi: 10.1192/bjo.2021.768

Aims. To summarise the tolerability profile following an infusion of methylene blue (MB), including subjective effects on mood and energy levels and haemodynamic changes, in patients with Bipolar Affective Disorder (BPAD).

Background. BPAD is associated with mitochondrial dysfunction and impaired cellular energy production. MB is proposed to enhance mitochondria function via rerouting electrons and intracellular reduction of oxidative stress, and is therefore a candidate compound for use as a probe to reveal alterations in brain oxygen metabolism in vivo in patients with $\mathrm{BPAD}$. Although there are reports of $\mathrm{MB}$ used as treatment for BPAD, the tolerability and subjective effects of a single IV dose in this population has not yet been defined.

Method. Using a single-blind, randomised, within-subject design, 7 patients with BPAD on stable pharmacological treatment and 6 healthy controls (HCs) received an infusion of $0.5 \mathrm{mg} / \mathrm{kg} \mathrm{MB}$ and a placebo glucose solution one week apart. Visual Analogue Scales (VAS) assessing 'Mood' and 'Energy' levels were completed by 11 participants, and blood pressure (BP), heart rate (HR) and any subsequent side effects were recorded before and after infusions. Result. A significant, albeit very small, effect of MB on 'Mood' levels relative to placebo was demonstrated, independent of groups (change relative to baseline: $5.5 \% \pm 11$ increase (placebo) vs $-1.6 \% \pm 9.5$ reduction $(\mathrm{MB}) ; \mathrm{p}=0.027)$. Although there was no effect of $\mathrm{MB}$ on energy levels in either group, there appeared to be a trend for a general group difference in 'Energy' levels across all trials, with lower ratings in BPAD patients $(\mathrm{p}=0.058)$.

There was a trend for significantly lower post-infusion HR relative to pre-infusion $(-6.4 \pm 8.8 \mathrm{bpm}, \mathrm{p}=0.07$. Diastolic BP was higher $(3.0 \pm 7.8 \mathrm{mmHg}, \mathrm{p}=0.039)$. These effects were independent of groups and drug. The most common side effect with $\mathrm{MB}$ was mild/moderate pain at infusion site $(n=10 / 13)$, resolving within median 32.5 minutes (IQR 6-102), and discoloured urine in $7 / 13$ subjects lasting median 44.5 hours (IQR 36-59). No difference in frequency of side effects reported between groups.

Conclusion. Although limited by small sample size, this tolerability analysis demonstrates a acceptable profile of effects of $\mathrm{MB}$ on subjective ratings and blood pressure, in both BPAD and HCs. Common side effects of discoloured urine and pain at infusion site are in line with previous reports in the literature. We observed a small effect of $\mathrm{MB}$ on mood ratings which could be related to the discomfort experienced during infusion.

\section{Virtual reality cognitive \& functional assessment in psychosis}

Sukhwinder Shergill*, Lilla Porffy, Gabriella Whomersley, Timea Szentgyorgyi, Elias Mouchlianitis and Joel Patchitt

Institute of Psychiatry, Psychology and Neuroscience, King's College London

${ }^{\star}$ Corresponding author.

doi: 10.1192/bjo.2021.769

Aims. To compare the MATRICS Consensus Cognitive Battery (MCCB) and a novel Virtual Reality (VR) task, called VStore, in assessing cognition and functional capacity (FC) in schizophrenia. We hypothesise that VStore reliably discriminates between patients and controls, correlates with the MCCB, and is welltolerated. Additionally, VStore is expected to strongly correlate with FC measures.

Background. Cognitive and functional deficits in schizophrenia have a major impact on everyday functioning of patients. The gold-standard cognitive assessment is the MCCB, while the USCD Performance-Based Skills Assessment (UPSA) is used to assess FC in this patient group. Neither of which are without limitations. For example, both take a long time to administer, and the MCCB alone cannot give clear indications of FC. We propose the use of a novel VR task to simultaneously measure cognition and FC in a single assessment. VStore is a shopping task, which involves a verbal learning task followed by buying items from a predetermined shopping list in a virtual minimarket.

Method. Ten patients with schizophrenia or schizoaffective disorder and ten age/gender-matched healthy controls recruited from South London, completed the following assessments: VStore, MCCB, UPSA \& Global Assessment of Functioning (GAF), and VR-Symptom Questionnaire (VRSQ); while controls only completed the VR task. To test whether VStore can differentiate between patients and controls we employed unpaired t-test. To explore associations between VStore Total Time, MCCB composite score and FC measures Pearson's r was used. Finally, mean differences between pre/post-VR symptoms scores were tested using paired t-test.

Result. There was a significant difference between patients and controls on the verbal learning task $(\mathrm{t} 16.38=-4.67, \mathrm{p}<.001)$, and total time spent completing the VR task (t11.41 $=2.67, \mathrm{p}=.023)$. In addition, VStore had a strong association with MCCB composite score $(\mathrm{r}=-.80, \mathrm{p}=.010)$. While both VStore $(\mathrm{r}=-.82, \mathrm{p}<001)$ and MCCB $(\mathrm{r}=.77, \mathrm{p}=.010)$ had significant correlation with the UPSA, only VStore had a significant association with the GAF $(\mathrm{r}=-.68, \mathrm{p}=.030)$. Finally, VStore appears to be well-tolerated, causing no measurable side effects in the VRSQ (Pre-VR Mean = $12.1[\mathrm{SD}=13.5]$, Post-VR Mean $=9.6[\mathrm{SD}=11.5], \mathrm{t} 9=0.49, \mathrm{p}>.05)$. 
Conclusion. Results suggest that VStore can discriminate between schizophrenia patients and healthy controls. In addition, VStore and MCCB seem to be strongly associated, suggesting that they tap into identical cognitive domains. VStore seems to be strongly correlated with FC, more so than the MCCB, and cause no measurable side effects. Taken together, this suggests that this novel VR task has the potential to reliably measure cognition and FC simultaneously.

\section{The emerging role of acceptance and commitment} therapy as a way to treat trauma and stressor related disorders

Gisela Simões* and Rita Silva

Department of Psychiatry and Mental Health of Baixo Vouga

Hospital Centre, EPE

${ }^{\star}$ Corresponding author.

doi: 10.1192/bjo.2021.770

Aims. The aim of this work is to gather and evaluate scientific evidence about the clinical effects of Acceptance and Commitment Therapy (ACT) in the treatment of patients with trauma-related Post-Traumatic Stress Disorder (PTSD).

Method. A literature search was conducted on PubMed platform, starting from the following MeSH terms: "Acceptance and Commitment Therapy", "Trauma and Stressor Related Disorders", "Psychological Trauma". Studies obtained were analysed, corresponding to investigations based on an adult population with trauma and stressor related disorders.

Result. The search provided 13 results, of which 12 met the defined criteria. Different types of studies with variable samples were considered, including randomised clinical trials, longitudinal observational studies, narrative reviews and an analysis of case reports.

Globally, ACT has been showing a crescent role in the treatment of individuals with trauma histories by enhancing positive outcomes and by being associated with greater psychological flexibility. It is increasingly considered to be well-suited to the treatment of trauma by targeting avoidance, coping strategies with emotional disengagement and persistent dissociation, aspects associated with greater PTSD symptom severity and related psychopathology.

Furthermore, research suggests that acceptance-based treatments are helpful in promoting emotional, behavioural, and neural changes in psychological disorders characterised by disgust, shame and guilt that commonly co-occur with PTSD.

Among the various exposure factors, we found a growing production of recent literature in which ACT has been applied in the context of oncology life-threatening settings, demonstrating significant improvements in symptoms and quality of life, as well as reductions in emotional disturbances, physical pain and traumatic responses.

However, little is known about implementation and results of ACT in situations of trauma and psychiatric comorbidities. Data suggest that, when applied to individuals with psychosis and history of trauma, there is an improvement in overall severity and anxiety symptoms, emotion regulation strategies and a greater sense of engagement in care; nevertheless, reduction of specific trauma symptoms remains controversial. More mention is made about the growth of literature evaluating the application of ACT as a conjunctial therapeutic method for trauma and simultaneous addictive disorders.

Conclusion. Overall, despite limited published research currently available, some evidence starts to support ACT's promising role as an effective psychotherapeutic approach to trauma and stressor related disorders. Its application in situations where organic diseases represent stress factors has been growing. Future research should focus on clarifying the role of ACT in psychiatric comorbidity scenarios, allowing this psychotherapy to help individuals find a meaningful and valuable life beyond trauma.

\section{Pharmacological interventions for improving cannabis use and psychosis in dual diagnosis: a systematic review \\ Arundeep Singh Bharj ${ }^{\star}$, Katy Jones and Musa Sami \\ University of Nottingham \\ ${ }^{\star}$ Corresponding author.}

doi: 10.1192/bjo.2021.771

Aims. Many patients with psychosis symptoms and schizophrenia use cannabis as a recreational drug. Patients who use cannabis respond differently to antipsychotic treatment compared to those who do not. Despite this, there is a lack of evidence, and therefore clinical guidance, pertaining to the best pharmacological treatment to improve psychosis or cannabis use in this population. This systematic review was carried out to assess the current evidence base regarding the most effective pharmacological treatment for patients with psychosis who also have a background of using cannabis. Our specific question was: in patients with a dual diagnosis of psychosis and cannabis use, which pharmacological interventions have the most efficacy in improving psychosis or reducing cannabis use?'.

Method. A search of EMBASE, PsychINFO, and MEDLINE(R) databases was carried out on September 30, 2020. Bibliographies of other studies were also searched for relevant articles. After exclusion of any articles which did not meet inclusion criteria for this review, eleven full texts remained; a qualitative analysis was carried out on these, but there was no meta-analysis. Only randomised control trials (RCTs) whose interventions and controls were pharmacological therapies, and which included patients with a background of cannabis use and psychosis, and which measured clinical outcomes, were included. Result. We found 11 articles which analysed 10 RCT studies $(\mathrm{n}=363)$ investigating risperidone, olanzapine, clozapine, haloperidol, ziprasidone and imipramine. 6/11 were double blind. The studies were small in size, varied in their methodology, exact inclusion criteria, exact outcomes, and all had a high risk of bias. Few significant findings were found. There is limited evidence for clozapine having anti-craving effect however whether this is associated with reduction in use remains to be demonstrated. We found no studies of adjunctive anticonvulsant agents, which are often used in psychotic disorders.

Conclusion. This review underlines the paucity of studies on which to make evidence-based decisions. No new studies have been undertaken since the last systematic review in this area in the last 7 years. Due to the lack of high-quality evidence found by this review, there remains a considerable need for interventional, high-quality RCTs in this comorbid patient group.

\section{Before the light fades, who blows the whistle? : a narrative review on sports dementia}

Olusegun Sodiya ${ }^{1 \star}$, Ovwigho Edafegwotu ${ }^{2}$ and Jide Jeje ${ }^{3}$

${ }^{1}$ Tees, Esk and Wear Valleys NHS Foundation Trust;

${ }^{2}$ Neuropsychiatric Hospital, Aro and ${ }^{3}$ Southern Health NHS

Foundation Trust

${ }^{*}$ Corresponding author.

doi: 10.1192/bjo.2021.772 